\title{
A removal kinetics approach for evaluation of economic cleaning protocols for pink guava puree fouling deposit
}

\begin{abstract}
Cleaning-in-place is a common process in food industries to maintain the hygiene of the processing system. In this paper, analysis on removal kinetics of the fouling deposit has shown promising results which can be used to improve CIP protocol. The removal kinetics analyses on the cleanability experiments of pink guava puree fouling deposit were done using the laboratory-scale cleaning test rig. The experiments' parameters were temperatures (35$\left.70{ }^{\circ} \mathrm{C}\right)$, Sodium hydroxide concentrations $(1.0-2.0 \mathrm{wt} \%)$ and fluid velocities $(0.6-1.5 \mathrm{~m} / \mathrm{s}$ at Reynolds numbers range of $\operatorname{Re}=4 \times 10^{4}$ to $18 \times 10^{4}$ ). Contact time of chemical wash and rinsing time were determined when the test object has reached a physically clean state. Two distinct cleaning stages: rapid and gradual were identified from the cleaning profiles and each stage has shown high effective removal rate at different cleaning conditions. The removal kinetics results were integrated with several types of cleaning protocols: 1) extreme cleaning; 2) optimal cleaning with water recirculation and 3) optimal cleaning. The cost analysis for every cleaning protocol has proved that the second cleaning protocol is more cost-effective compared to the other cleaning protocols.
\end{abstract}

Keyword: Removal kinetics; Pink guava puree fouling deposits; Cleaning protocol; CIP cost 
